\title{
EDITORIAL
}

\section{MALARIA DIAGNOSIS}

Malaria remains the most significant parasitic disease affecting man. Prompt and accurate diagnosis of malaria is the key to cost effective management (1). Since the identification of Plasmodium parasites in human blood in 1880, the diagnosis of malaria has remained a hot bed of scientific discussion. The currently available diagnostic techniques include microscopy, rapid diagnostic tests, polymerase chain reaction (PCR), automated flow cytometry, clinical and presumptive diagnosis. These diagnostic techniques each have inherent limitations that have not been fully resolved.

Microscopy is the oldest technique and is referred to as the "gold standard". There are two forms of microscopic techniques, the Romanosky stains and the fluorescent technique. Several stains that have been used for staining the thick and thin blood films include Giemsa, Field, Jaswant Singh Bhattacharji, Diff Quick, May-Guanwald, panoptic, and Leishman and Wright. Of these, the most commonly used are Giemsa and Field stain, and of the two Giemsa is the most preferred. Field stain requires a shorter staining time and is cheaper, but it has limitations in delineating the features of Plasmodium for species characteristics and it also deteriorates rapidly so is not suitable if the slides are to be stored for long. With the Giemsa stain technique, there are several factors that can impact the quality of blood slides such as the source and quality of slides and stain, the ph of the buffer used, quantity of blood used, and length of the staining period. These subtle specificities are critical for species identification, in the turn-around for slide results, and for the long term storage of blood slides. There is another drawback with microscopy, and that is its subjective nature. One must rely on the competence of the microscopist. As a result, microscopy is referred to as the "imperfect reference" (2). No international acceptable standards are available to reference about slide preparation, stain preparation, staining technique, slide reading paradigms, results reporting format, or for who qualifies as a microscopist to be referred to as an "expert microscopist." Such standards are difficult to establish because, depending upon the circumstances, one way of performing microscopy may be more appropriate than others, based on the anticipated outcome and use of the data generated (i.e., the occurrence of false positive can ruin a prophylactic drug or vaccine trial) (2). When the results of trials are published, it is essential that the microscopy techniques used and competency of the microscopist be reported in order to enable readers to determine the validity of the study outcome; however, this is rarely done.

Despite its limitations, the microscopy technique is the only diagnostic technique that can provide accurate species identification and quantification of the parasite load. The confines of this technique has led to the current drive to set up centres of excellence for microscopy to help formulate standards for performing microscopy, and for training and proficiency testing of microscopists. There are also efforts to improve microscopy by the use of grid lenses as these can easily be employed to standardise the method of scanning fields on a slide and also for parasite quantification. The use of grid lenses is not widely practiced, and still requires more validation.

The fluorescent microscopy technique, which involves staining the blood film with fluorescent dye (usually acridine dye) or with acridine dye in quantitative buffy coat (QBC), has been used to identify P. falciparum. This method is an alternative to non-flourescent microscopy in malaria endemic areas $(3,4)$. However, QBC is expensive, it cannot be used for species identification and parasite load estimation, and it is currently not available commercially. An infrequently used alternative to acridine dye is Rhodamine 123 (R123), but its uptake by the red blood cells is affected by some antimalarial drugs. The role of fluorescent techniques for malaria diagnosis remains limited.

The last decade has seen the introduction of rapid diagnostic techniques (RDT) to improve the speed, and to simplify, the diagnosis of malaria. These techniques are based on the capture of Plasmodium specific antigens in finger prick blood samples, and are marketed as immunochromatographic kits designed for single sample use. The antigens commonly targeted are histidine rich protein 2 (HRP2), a water soluble species-specific protein that is produced by asexual stages and young gametocytes of Plasmodium species, and parasite lactate dehydrogenase ( $\mathrm{pLDH})$, a soluble glycolytic enzyme that is expressed at high levels in asexual stages of malaria parasites. The RDT kits are available under different names depending on the manufacturing company. The techniques are easy to learn and to implement, and so they are useful in field situations (i.e. epidemics), but they are more expensive compared to microscopy. One disadvantage of the RDT is that sensitivity and specificity declines rapidly in low parasite densities $(<100$ parasites per $(\mu \mathrm{l})(3)$. Another is the persistence of these antigens (especially HRP2 which may persist for $14-21$ days) even if the parasites have been cleared; hence their use may result in a false positive. These techniques are also species specific; hence they are not useful in the case of mixed infections. They can also cross react with other auto-antibodies, such as rheumatoid factor, resulting in false positives. And, they cannot be used for parasite load estimation.

The last decade has seen an expansion in the use of PCR to diagnose diseases including malaria. The technique detects circulating parasite DNA, so sensitivity 
and specificity is $100 \%$ even at very low parasite densities (such as 5 parasites per $\mu \mathrm{l}$ ). However, because it is a very expensive technique, requires extensive training, and the results take longer, its role in clinical practice has not been evaluated. Even with the development of quantitative PCR, the use of this technique for parasite load estimation has not been elucidated. However, it remains a powerful diagnostic tool for research purposes.

The automated flow cytometry technique is based on the fluorescence of malaria pigments in white blood cells. This technique cannot be used for species identification or parasite load estimation. It is dependent on the pigment load in circulation; thus, there is a greater likelihood that early infections will be missed. The persistence of pigments in some white blood cells, such as monocytes, may lead to false positive cases. Additionally, this technique requires very expensive instrumentation, and so is out of reach for most health facilities in resource poor countries. Its role in the clinical setting has not been evaluated.

The clinical algorithm is the most common diagnostic technique used in malaria endemic areas, especially in peripheral health facilities where other diagnostic methods are not available. It is based on the presumption that the presence of fever equals malaria; thus, it is quite inaccurate, even when the most stringent clinical algorithm is applied (5). It is used in malaria endemic areas for public health purposes to avert presumed malaria related deaths. It is likely to result in over treatment, with a resultant increase in drug pressure. Due to resource constraints, this is the "diagnostic technique" of last resort when none other is available.

Presumptive diagnostic technique is a public health tool that hinges on the fact that a subset of the population is at increased risk of malaria infection and poor outcome from the disease. The target population is more often than not at increased risk of poor outcome due to the lack of required medical amenities which enable prompt and accurate diagnosis and treatment. This diagnosis technique is currently restricted to pregnant women and children under the age of five years in malaria endemic areas $(6,7)$. The target populations are intermittently given a full course of antimalarial treatment to treat presumed infection, be it clinical or sub-clinical, and depending on the drug used, may also be used to provide a prophylactic effect.
Microscopy in the best of hands remains the 'gold standard' for malaria diagnosis and the other techniques currently available are there to supplement rather than replace it. As more validated diagnostic techniques become available, several factors are likely to influence the choice of a diagnostic test to be used: the level of malaria endemicity, first line treatment, prevalence and type of drug resistance, geographical accessibility, available health infrastructure, social and economic factors, and the availability of diagnostic tools. While we look forward to the development of more robust, inexpensive, and less subjective techniques with shorter turn-around times, there is an urgent need to develop centres of excellence for microscopy to help establish internationally accepted standards of microscopy and training of microscopists now.

B. R. Ogutu, MBChB, MMed, PhD, Senior Research Officer, Walter Reed Project-Centre for Clinical Research, Kenya Medical Research Institute, P.O. Box 54, Kisumu, Kenya

\section{REFERENCES}

1. WHO (2000) New Perspectives: Malaria Diganosis. WHO/ MAL/2000. 1091.

2. Ohrt, C., Purnomo, Sutamihardja M. A., Tang, D. and Kain K. C. Impact of microscopy error on estimates of protective efficacy in malaria-prevention trials. J. Infect Dis. 2002; 186:540-546.

3. Olaleye, B. O., Williams, L. A., D'Alessandro, U. et al. Clinical predictors of malaria in Gambian children with fever or a history of fever. Trans. R. Soc. Trop. Med. Hyg. 1998; 92:300-304.

4. Lema, O. E., Carter, J. Y., Nagelkerke, N. et al. Comparison of five methods of malaria detection in the outpatient setting. Amer. J. Trop. Med. Hyg. 1999; 60:177-182.

5. Lowe, B. S., Jeffa, N. K., New, L. et al. Acridine orange fluorescence techniques as alternatives to traditional Giemsa staining for the diagnosis of malaria in developing countries. Trans. R. Soc. Trop. Med. Hyg. 1996; 90:34-36.

6. Shulman, C. E., Dorman, E. K., Cutts, F. et al. Intermittent sulphadoxine-pyrimethamine to prevent severe anaemia secondary to malaria in pregnancy: a randomised placebocontrolled trial. Lancet. 1999; 353(9153):632-636.

7. Schellenberg, D., Menendez, C., Kahigwa, E. et al. Intermittent treatment for malaria and anaemia control at time of routine vaccinations in Tanzanian infants: a randomised, placebo-controlled trial. Lancet. 2001; 1:358(9283):762-763. 